\title{
The Hemodynamic Impact of Unstable Coronary Plaques - Do We Have the Evidence?
}

\section{Theodora Benedek}

Laboratory of Advanced Research in Multimodality Cardiac Imaging, University of Medicine and Pharmacy, Tîrgu Mureș, Romania

In the last years, estimation of the functional relevance of a coronary artery stenosis has raised an increased interest, and new imaging-derived parameters have been proposed as promising tools for this application. Invasive FFR represents nowadays the gold standard for the estimation of lesion significance, while noninvasive CT-FFR has been proposed as an alternative technique with good sensitivity and positive predictive value for predicting an FFR $<0.8$, which is the reference value for classifying a lesion as significant.

Transluminal attenuation gradient (TAG) by computed tomography angiography (CTA) has been suggested to represent a faster alternative to invasive FFR or to noninvasive FFR-CT for assessing lesion-specific ischemia. In a recent meta-analysis, corrected coronary opacification decrease was an independent predictor of major adverse cardiac events (MACE) in patients with coronary artery stenosis, adding long term prognostic value over clinical predictors or classical biomarkers. ${ }^{1}$ The usefulness of TAG in plaque characterization is especially obvious in highly calcified lesions, in which the presence of calcium precludes good quality imaging of the coronary plaques. ${ }^{2}$ In these cases, TAG can significantly contribute to the reclassification of stenosis degree and to choosing the most appropriate therapeutic strategy.

It has been proposed that noninvasive CTA-based lesion-specific ischemia will represent the gatekeeper to the cardiac catheterization laboratory in a close future, as soon as the technique and its application will be validated by large trials. ${ }^{3}$ TAG, myocardial perfusion, and CT and CT-FFR are the most common studied imaging tools for the assessment of lesion-specific ischemia, because ischemia-guided revascularization can significantly improve patient outcomes. ${ }^{4}$ CT-FFR proved superior to TAG, CTAevaluated stenosis degree or the combination of the two in terms of diagnostic accuracy in the DISCOVER-FLOW and DeFACTO studies. ${ }^{5}$

However, little is known in present about the role of plaque vulnerability degree in increasing the functional significance of a stenosis. Morphological and functional quantitative plaque assessment using CTA can provide us relevant information on the presence of vulnerability markers inside an atheromatous plaque, with the concomitant calculation of parameters reflecting functional significance such as TAG, myocardial perfusion, or CTFFR. ${ }^{6}$ A study on a limited number of patients, published in this number of JCE by Orzan et al. shows a good correlation between noninvasive TAG and invasive FFR ( $r=0.7$, $\mathrm{p}=0.01$ ) in the culprit lesions of patients with acute coronary syndromes, at the same time proving that the presence of vulnerability markers inside a plaque (an increased amount of necrotic core or a higher plaque burden) are associated with higher TAG values. ${ }^{7}$ These findings suggest that the presence of vulnerability features inside a coronary plaque could increase the functional significance of a coronary lesion, and that vulnerable plaques could have a stronger impact on the myocardial ischemia than nonvulnerable ones with the same morphological severity.

However, the value of this interesting study is significantly altered by the low number of cases enrolled, being actually a pilot study. This could also represent an explanation for the lack of statistical significance in many of 
the associations studied, as the number of cases was not sufficient to generate statistical power. A future extension of the study should provide not only a higher number of patients for data validation, but also more applications of CTA such as myocardial perfusion imaging or shear stress assessment at the site of vulnerable lesions, in order to explore all the potential inter-relations between vulnerable plaques and associated ischemia. This could be particularly important as there are no large studies published so far to elucidate the hypothesis that vulnerable plaques can have an increased impact on the distal blood flow, causing more severe ischemia as compared with stable plaques.

The role of inflammation in the complex process of atheromatous plaque formation is also not negligible, and the authors mention the potential role of inflammation and local shear stress in plaque progression. Taking into consideration that an augmented inflammation can lead to plaque vulnerabilization, at the same time altering the local coronary blood flow and shear stress distal to the plaque, an extension of this study could also address the relation between the elevation of inflammatory biomarkers, the alteration of coronary flow, and the resulting myocardial ischemia. In this way, the study could provide the necessary evidence to demonstrate that plaque vulnerabilization can lead to an increased functional significance of the lesion, reflected in a lower coronary perfusion distal to the lesion and a more severe lesion-specific ischemia.

\section{CONFLICT OF INTEREST}

Nothing to disclose.

\section{REFERENCES}

1. Benz DC, Mikulici F, Grani C, et al. Long-term outcome prediction by functional parameters derived from coronary computed tomography angiography. Int J Cardiol. 2017 May 24. pii: S0167-5273(17)30601-0. doi: 10.1016/j.ijcard.2017.05.083. [Epub ahead of print]

2. Fengfeng Y, Jie D, Wei W, et al. Evaluation of stenosis severity of coronary calcified lesions using transluminal attenuationgradient: clinical application of 320-row volume CT. Minerva Med. 2017 Mar 1. doi: 10.23736/So0264806.17.04862-5. [Epub ahead of print]

3. Hecht HS, Narula J, Fearon WF. Fractional Flow Reserve and Coronary Computed Tomographic Angiography: A Review and Critical Analysis. Circ Res. 2016;119(2):300-16. doi: 10.1161/ CIRCRESAHA.116.307914.

4. Koo HJ, Yang DH, Kim YH, et al. CT-based myocardial ischemia evaluation: quantitative angiography, transluminal attenuationgradient, myocardial perfusion, and CT-derived fractional flow reserve. Int J Cardiovasc Imaging. 2016;321:119. doi: 10.1007/s10554-015-0825-5. Epub 2015 Dec 14.

5. Nakanishi R, Matsumoto S, Alani A, et al. Diagnostic performance of transluminal attenuation gradient and fractional flow reserve by coronary computed tomographic angiography (FFR(CT)) compared to invasive FFR: a subgroup analysis from the DISCOVER-FLOW and DeFACTO studies. Int J Cardiovasc Imaging. 2015;31:1251-9. doi: 10.1007/ s10554-015-0666-2.

6. Tesche C, Cecco CN, Caruso D, et al. Coronary CT angiography derived morphological and functional quantitative plaque markers correlated with invasive fractional flow reserve for detecting hemodynamically significant stenosis. J Cardiovasc Comput Tomogr. 2016;10:199-206. doi: 10.1016/j. jcct.2016.03.002.

7. Orzan M, Dobra M, Chitu M. A comparative preliminary study on CT contrast attenuation gradient versus invasive FFR in patients with unstable angina. J Cardiovasc Emerg. 2017;2:... 